\title{
Brief encounter: UTI triggered by vaginal microbiota
}

New data show that recurrent UTI can be triggered by transient exposure to vaginal bacteria, specifically Gardnerella vaginalis. These results suggest that UTI pathogenesis can be driven by short-term urinary tract exposure to vaginal microbiota, which are not themselves traditionally considered uropathogenic. Thus, targeting specific components of the vaginal microbiota could be an effective method of treating recurrent UTI.

Gilbert and colleagues investigated the effects of vaginal bacteria exposure on the urinary tract using a mouse model of intracellular Escherichia coli reservoirs. G. vaginalis (which contributes to bacterial vaginosis) exposure represented dysbiotic vaginal microbiota and Lactobacillus crispatus exposure represented normal vaginal microbiota in this experimental context.

Transient urinary tract exposure to $G$. vaginalis induced $E$. coli emergence into urine despite this pathogen not colonizing the urinary tract. Detection of E. coli in the urine was increased after two exposures to $G$. vaginalis even if the exposures were 2 weeks apart.
Moreover, these mice had neutrophilic infiltrates in their urine, indicating that emerging $E$. coli constituted active recurrent UTI, and urothelial cells were also identified in their urine sediments. Furthermore, mice that experienced G. vaginalis-induced recurrent UTI were those that had prolonged and severe primary infection after E. coli inoculation. By contrast, exposure to L. crispatus or heat-killed $G$. vaginalis did not induce $E$. coli emergence. Analysis of the bladders of mice exposed to $G$. vaginalis using scanning electron microscopy (SEM) showed areas of exfoliation and detachment of individual cells, whether or not these mice had latent $E$. coli reservoirs, whereas bladders of mice exposed to L. crispatus did not exhibit exfoliation. Furthermore, SEM revealed membrane protrusions and blebbing in bladder cells exposed to $G$. vaginalis, with positive TUNEL assay results and cleaved CASP3 staining, indicating that these bacteria can induce apoptosis.

The kidneys of mice exposed to G. vaginalis showed increased expression of markers of kidney injury, an effect that was blocked by treatment with an IL-1 receptor agonist. G. vaginalis exposure also increased the susceptibility of kidneys to E. coli infection and resulted in kidney abscesses and splenomegaly in $6 \%$ of mice that had E. coli reservoirs. Analysis of the spleens of mice with abscessed kidneys showed G. vaginalis and E. coli could both cause systemic infection.

These data provide evidence of a direct role for transient vaginal microbiota exposure in UTI pathogenesis and that exposure to vaginal bacteria can result in kidney damage. Targeting specific bacteria with the vaginal microbiota could prevent recurrent UTI and subsequent pyelonephritis.

Louise Stone

ORIGINAL ARTICLE Gilbert, N. M. et al. Transient microbiota exposures activate dormant Escherichia coli infection in the bladder and drive severe outcomes of recurrent disease. PLOS Pathogens http://dx.doi.org/10.1371/journal.ppat.1006238 (2017) 